\title{
Die UN-Behindertenrechtskonvention: Feste Größe in einem psychiatriepolitischen Schlingerkurs?
}

\author{
The UN Convention on the Rights of Persons with Disabilities - \\ Gaining a Foothold in Psychiatry's Political Seesaw?
}

Autor

Institut

\author{
Raoul Borbé
}

Zentrum für Psychiatrie Südwürttemberg, Abt. für Allgemeinpsychiatrie Weissenau und Psychiatrie I der Universität Ulm

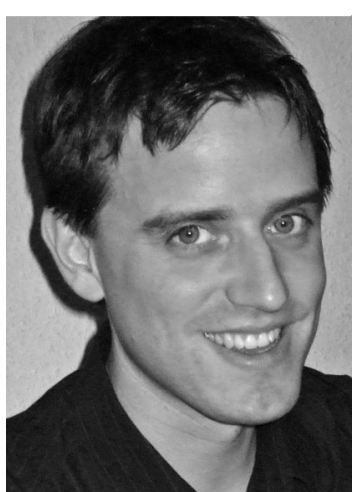

Dr. med. Raoul Borbé

\section{Bibliografie}

Dol http://dx.doi.org/ 10.1055/s-0030-1266111 Psychiat Prax 2011; 38: 215-217

(c) Georg Thieme Verlag KG Stuttgart · New York. ISSN 0303-4259

Korrespondenzadresse Dr. med. Raoul Borbé Zentrum für Psychiatrie Südwürttemberg, Abt. für Allgemeinpsychiatrie Weissenau und Psychiatrie I der Universität Ulm Weingartshofer Straße 2 88214 Ravensburg raoul.borbe@zfp-zentrum.de
Psychiatriepolitik war von jeher ein sensibles Thema und immer wieder prägend für die Versorgung psychisch Kranker - im positiven wie im negativen Sinne. Auch in den letzten Jahren gab es politische Entscheidungen, die die Versorgung psychisch Kranker nachhaltig beeinflussen werden. Noch ist aber völlig unklar, wie die Versorgungslandschaft in 10 Jahren aussehen wird. Im Gegensatz zum Bericht der vor mittlerweile 40 Jahren einberufenen Enquetekommission des Deutschen Bundestages zur Lage der Psychiatrie in der Bundesrepublik Deutschland fehlt der Politik heute eine übergeordnete Vision, wie die Versorgung psychisch Kranker verbessert werden soll. Stattdessen werden mit aktuellen psychiatriepolitischen Entscheidungen meist Partialinteressen verfolgt, häufig mit konzentriertem Blick auf ökonomische Parameter. Zur besseren Veranschaulichung dieser Thesen sollen im Folgenden 3 wesentliche politische Entscheidungen der letzten 2 Jahre näher beleuchtet und diskutiert werden.

\section{Ratifizierung der UN-Behindertenrechts- konvention (UN-BRK)}

Die UN-BRK kann jetzt schon als weiterer Markstein einer langjährigen Entwicklung hin zu einer patientenorientierten, die subjektiven Bedürfnisse der Patienten beachtenden Grundhaltung gesehen werden [1]. Zu den Grundannahmen der UN-BRK zählen die Entstehung von Behinderung aus der Wechselwirkung zwischen Menschen mit Beeinträchtigungen und einstellungs- und umweltbedingten Barrieren, die Vielfalt der Menschen mit Behinderungen, die Wichtigkeit von Autonomie und Unabhängigkeit für Menschen mit Behinderungen und die Wichtigkeit des vollen Zugangs zur physischen, sozialen, wirtschaftlichen und kulturellen Umwelt, zu Gesundheit und Bildung sowie zu Information und Kommunikation für Menschen mit Behinderungen.

Neben einer grundsätzlichen Betonung der Menschenrechte und Grundfreiheiten, auf die jeder Mensch ohne Unterschied Anspruch hat, ist die UN-BRK daher auch durchzogen vom Gedanken der sozialen Inklusion, was sich durch den Einbezug von Menschenrechten der 2. Generation, die Teilhabe- und Solidarrechte umfassen, manifestiert. Zu den wichtigsten Forderungen gehören daher u.a. Gleichberechtigung und Nichtdiskriminierung, Bewusstseinsbildung für Menschen mit Behinderungen, Zugänglichkeit zur physischen Umwelt, zu Transportmitteln, zu Information und Kommunikation, gleiche Anerkennung vor dem Recht, Zugang zur Justiz, Freiheit und Sicherheit der Person, Arbeit und Beschäftigung, Teilhabe am politischen und öffentlichen Leben und am kulturellen Leben.

Der Ratifizierung der UN-BRK durch die Bundesrepublik Deutschland im März 2009, folgte im Oktober 2009 im Koalitionsvertrag von Union und FDP die Ankündigung eines „Aktionsplans zur Umsetzung der Behindertenrechtskonvention der Vereinten Nationen". Das Monitoring der Umsetzung der UN-BRK war bereits im Jahr 2008 an das Deutsche Institut für Menschenrechte vergeben worden [2]. Der 1. Bericht der Bundesrepublik Deutschland an die Vereinten Nationen muss nun im Frühjahr 2011 erfolgen. In den nächsten Jahren wird dieser Bericht möglicherweise eine wichtige Richtschnur für die Psychiatriepolitik. Die UN-BRK könnte in diesem Sinne als Fortsetzung der Psychiatrie-Enquête und der Empfehlungen der Expertenkommission aus dem Jahre 1988 gesehen werden. Mit der Forderung nach gleichberechtigter sozialer Inklusion nimmt sie die seit Mitte des letzten Jahrzehnts monierten Fehlentwicklungen im Umbau der psychiatrischen Versorgungslandschaft durch Transinstitutionalisierung und soziale Exklusion $[3,4]$ auf. 
Viele bereits vorhandene und in Planung befindliche Projekte erhalten durch die UN-BRK einen höheren Stellenwert. Neben der Antistigma-Arbeit ist es vor allem der von der Aktion Psychisch Kranke e.V. (APK) entwickelte personenzentrierte Ansatz [5], der ein selbstbestimmt-mitwirkendes Patientenbild zur Grundlage hat und sich deckungsgleich in den Grundtenor der UN-BRK einfügt. Nicht umsonst verweist die APK in ihrer Stellungnahme zur Ratifizierung der UN-BRK auf die Notwendigkeit die Gestaltungsaufgaben für die Selbstverwaltung, die im SGB IX formuliert werden, nun auch umzusetzen [6]. Als Problem wird in diesem Zusammenhang die Fragmentierung des deutschen Gesundheits-, Rehabilitations- und Sozialleistungssystems gesehen, die aber durch die nächste, hier zu beleuchtende politische Entscheidung erst einmal zementiert wird.

\section{Krankenhausfinanzierungsreformgesetz \\ $\nabla$}

Das Krankenhausfinanzierungsreformgesetz (KHRG) wurde vom Deutschen Bundestag 2009 mit dem Ziel verabschiedet, für die stationäre Psychiatrie und die Psychosomatik ein neues Entgeltsystem einzuführen [7]. Wie das System in Zukunft aussehen wird, mag man nach ersten aktuellen Publikationen zur Sinnhaftigkeit der jetzt betriebenen Leistungserfassung noch nicht sagen [8]. Klar ist aber, dass durch die Abkopplung der ambulanten Versorgung im Entwicklungs- und Einführungsprozess des neuen Entgeltsystems wichtige Zeit verloren geht, um die Trennung des stationären vom ambulanten Sektor zu überwinden. Dabei existieren bereits unterschiedliche Ansätze, bisher nur in Modellprojekten, die genau dies tun. Zu nennen wären das Regionalbudget $[9,10]$ und sog. IV-Verträge. Die integrierte Versorgung nach $§ 140$ SGB V wurde 2004 vom Gesetzgeber eingeführt, mit dem Ziel, Integrationsverträge zwischen Leistungserbringern und Krankenkassen zu ermöglichen. Auch in der Psychiatrie gibt es mittlerweile eine Reihe von IV-Verträgen, die teils sehr positive Ergebnisse erbrachten [11].

\section{Arzneimittelmarktneuordnungsgesetz \\ $\nabla$}

Ergänzend dazu wurde durch das AMNOG, das Arzneimittelmarkt-Neuordnungsgesetz aus dem Jahre 2010 [12] nun auch Pharmafirmen die Möglichkeit eingeräumt, IV-Verträge mit Krankenkassen abschließen zu können. Im November 2010 wurde das bereits durch die AOK Niedersachsen und den Pharmakonzern Janssen-Cilag in die Realität umgesetzt. Zwischengeschaltet wurden, möglicherweise um Bedenken zu zerstreuen, eine Tochtergesellschaft von Janssen-Cilag, die I3G GmbH, die die Budgetverantwortung übernehmen soll und Care4S, eine Gesellschaft, die für das operative Geschäft zuständig sein wird. Wegen naheliegender Interessenkonflikte wurde dieses Gesetz von Anfang an durch verschiedene Verbände, u.a. die Deutsche Gesellschaft für Psychiatrie, Psychotherapie und Nervenheilkunde DGPPN, sehr kritisch beurteilt [13].

Allerdings wäre es zu einfach, die Fehlentwicklungen nur einer verfehlten Psychiatriepolitik anzulasten. Auch ein sich verändernder Mainstream in der psychiatrischen Forschung trägt zu dem geringeren Stellenwert bei, der der personenzentrierten Versorgung nicht nur von Politikerseite beigemessen wird. Ana$\log$ zur Biologisierung der Psychiatrie liegt der Anteil von Forschungsarbeiten bei schizophrenen Patienten, die die subjektive
Sicht der Patienten in den Mittelpunkt stellen, bei $2 \%$ [14]. Personenzentrierung spielt in der „decade of the brain“ also nur eine untergeordnete Rolle in der psychiatrischen Forschung. Ebenso fehlt es, gerade in Deutschland - nicht zuletzt wegen der Zergliederung des Versorgungssystems -, an validen Zahlen aus der Versorgungsforschung, wobei auch international ein hoher Nachholbedarf gesehen wird $[15,16]$.

Und hier kommt wieder die UN-BRK ins Spiel, die uns eine Vielzahl von Hinweisen und Vorgaben gibt, wie ein Staat, eine Gesellschaft, aber auch die ganz praktisch an der Versorgung psychisch Kranker Beteiligten soziale Inklusion gestalten können [17].

Die UN-BRK wird Auswirkungen auf die Organisation von sozialen Dienstleistungen und deren Anbieter haben. Stichworte wie Ex-In, user-involvement, user-led-research und Inklusionsagenturen sind alles schon vorhandene, wohlklingende Ansätze zum Einbezug der Betroffenen in die Planung und Gestaltung von Versorgungsstrukturen. Solange es in Deutschland aber nicht einmal eine flächendeckende Versorgungsverpflichtung der gemeindepsychiatrischen Verbünde gibt, solange ein psychisch Kranker durch einen gesetzlichen Betreuer regelhaft von einem badenwürttembergischen Landkreis in eine geschlossene Heimeinrichtung in Bayern mit mehreren 100 Betten transferiert werden kann, solange kann alles andere nur Stückwerk sein und damit nicht im Sinne der UN-BRK (u. a. §§ 25 - Gesundheit und 26 -Habilitation und Rehabilitation).

Die Umsetzung der UN-BRK muss deshalb überwacht werden: das gilt nicht nur für die Politik, die das dem Deutschen Institut für Menschenrechte als Monitoringstelle überantwortet hat, sondern gerade auch für die Arbeit vor Ort. Der Einstieg könnte z.B. durch den regionalen Ausbau und die Vernetzung unabhängiger Beschwerdestellen, Ethikkommissionen sowie staatlicher Besuchskommission erfolgen, die in Einzelfällen Einsprüche von Patienten oder Angehörigen zeitnah überprüfen und rückmelden sowie nach der Bündelung von Einzelfällen regelmäßig Empfehlungen für die zu entwickelnde Systemsteuerung aussprechen. Schließlich fordert die UN-BRK aber auch die Betroffenen, ohne deren Eigenverantwortung (und die aktive Solidarität der Zivilgesellschaft) die soziale Inklusion nicht $\mathrm{zu}$ verwirklichen ist $[18,19]$.

Insofern muss der angekündigte Aktionsplan der Bundesregierung zur Umsetzung der UN-BRK kritisch begleitet werden, gerade in Zeiten der Ressourcenverknappung und Verteuerung von Gesundheitsleistungen, denn die Politik darf nicht „Selbstverantwortung und -hilfe als Lückenbüßer für leere Kassen und Ratlosigkeit begreifen“ [18]. Dies kann in der Praxis aber keine Ausrede dafür sein, Maßnahmen vor Ort nicht zu prüfen oder umzusetzen. Viele Hürden sind auch in unseren Köpfen und ohne finanziellen Aufwand beseitigbar und um mit Peter Bartlett zu sprechen: „many of the adjustments required will thus not require a seismic shift in the way we think of law and mental disability“ [20]. Für fast jede in der UN-BRK formulierte Forderung gibt es zumal unter Einbezug der internationalen Perspektive - bereits jetzt erfolgreiche Lösungsansätze und Versorgungsmodelle. Wir müssen diese nur umsetzen.

\section{Literatur}

1 UN-Behindertenrechtskonvention (amtliche deutsche Übersetzung). www.un.org/Depts/german/uebereinkommen/ar61106-dbgbl.pdf

2 Aichele $V$. Die unabhängige Monitoring-Stelle zur UN-Behindertenrechtskonvention in Deutschland: Hintergrund, Ausrichtung, Wirkungszusammenhang. Zeitschrift für Inklusion Nr. 2 / 2010 
3 Priebe S, Badesconyi A, Fioritti A et al. Reinstitutionalisation in mental health care: comparison of data in service provision from six European countries. Brit Med J 2005; 330: 123-126

4 Eikelmann B, Zacharias-Eikelmann B, Richter D et al. Integration psychisch Kranker: Ziel ist Teilnahme am „wirklichen“ Leben. Dtsch Arztebl 2005; 102: B-929

5 Kunze H. Die Idee des personenzentrierten Ansatzes. In: Schmidt-Zadel R, Kunze H, Aktion Psychisch Kranke. Die Zukunft hat begonnen. Personenzentrierte Hilfen - Erfahrungen und Perspektiven. Bonn: Psychiatrie-Verlag; 2004: 17-29

6 Aktion Psychisch Kranke e.V. APK. Aus der Stellungnahme der Aktion Psychisch Kranke. Psychosoziale Umschau 2009; 24: 35-36

7 Bundesgesetzblatt. Gesetz zum ordnungspolitischen Rahmen der Krankenhausfinanzierung ab dem Jahr 2009 (Krankenhausfinanzierungsreformgesetz - KHRG). Bundesgesetzblatt; Jahrgang 2009, Teil I Nr. 15: 534-549

8 Belling R. Psych-Pretest offenbart Diskussionsbedarf. f\&w 2011; 28: 61-65

9 Deister A, Zeichner D, Witt $T$ et al. Veränderung der psychiatrischen Versorgung durch ein Regionales Budget - Ergebnisse eines Modellprojektes in Schleswig-Holstein. Psychiat Prax 2010; 37: 335-342

10 König HH, Heinrich S, Heider D et al. The regional psychiatry budget (RPB): a model for a new payment system of hospital based mental health care services. Psychiat Prax 2010; 37: 34-42

11 Lambert M, Bock T, Schöttle D et al. Assertive community treatment as part of integrated care versus standard care: a 12-month trial in patients with first- and multiple-episode schizophrenia spectrum disor- ders treated with quetiapine immediate release (ACCESS trial). J Clin Psychiatry 2010; 71: 1313-1323

12 Bundesgesetzblatt. Gesetz zur Neuordnung des Arzneimittelmarktes in der gesetzlichen Krankenversicherung (Arzneimittelmarktneuordnungsgesetz - AMNOG). Bundesgesetzblatt; Jahrgang 2010, Teil I Nr. 67: 2262-2277

13 Rieser S. IV-VERTRAG SCHIZOPHRENIE: Pharmatochter ist Vertragspartner. Dt Ärzteblatt 2011; 108: A146-A148

14 Calton T, Cheetham A, D'Silva K et al. International Schizophrenia Research and the concept of patient-centredness: an anlysis over two decades. Int J Soc Psych 2009; 55: 157-169

15 Cobigo V, Stuart H. Social inclusion and mental health. Curr Opin Psychiatry 2010; 23: 453-457

16 Riedel-Heller SG. Research in social psychiatry - addressing future challenges of health- and social systems. Neuropsychiatr 2009; 23: 249252

17 Frühwald S, Karner A, Seyringer ME et al. Quality assurance of take-over from in-patient to out-patient care: experiences in Lower Austria. Neuropsychiatr 2010; 24: 195-199

18 Wienberg G. Gemeindepsychiatrie heute - Erreichtes, aktuelle Herausforderungen und Perspektiven. Sozialpsychiatrische Informationen 2008; 38: 2-13

19 Richter D. Evaluation of a residential care and supported housing program in the regional association of Westphalia-Lippe. Psychiat Prax 2010; 37: 127-133

20 Bartlett $P$. The United Nations Convention on the Rights of Persons with Disabilities and the future of mental health law. Psychiatry 2009; 8: 496-498 